\title{
THE DIFFERENTIABILITY OF TRANSITION FUNCTIONS ${ }^{1}$
}

\author{
BY DONALD ORNSTEIN \\ Communicated by J. L. Doob, October 6, 1959
}

In this paper we prove that the transition functions of a denumerable Markoff chain are differentiable or equivalently: Given a matrix of real valued functions $P_{i j}(t)(i, j=1,2, \cdots) 0 \leqq t<\infty$ satisfying

$$
P_{i j}(t) \text { is non-negative and continuous, }
$$

$$
\begin{aligned}
P_{i j}(0) & =\left\{\begin{array}{l}
1 \text { if } i=j, \\
0 \text { if } i \neq j,
\end{array}\right. \\
P_{i j}\left(t_{1}+t_{2}\right) & =\sum_{k=1}^{\infty} P_{i k}\left(t_{1}\right) P_{k j}\left(t_{2}\right), \\
\sum_{j=1}^{\infty} P_{i j}(t) & =1 .^{2}
\end{aligned}
$$

Our theorem is that $P_{i j}(t)$ has a finite continuous derivative for all $t>0$.

This result was conjectured by Kolmogoroff in [4].

Doob showed [3] that $P_{i j}(t)$ has a right hand derivative (possibly infinite) at $t=0$ and Kolmogoroff showed [4] that this derivative is finite if $i \neq j$, (if $i=j$ there are examples where it is infinite). Austin $[1 ; 2]$ showed that that $P_{i j}(t)$ has a finite continuous derivative for $t>0$ if either $P_{i i}(t)$ or $P_{j j}(t)$ has a finite derivative at 0 .

We will now give the proof ${ }^{3}$ of our theorem. We will think of the matrices $\left\{P_{i j}(t)\right\}$ as transformations on sequences in such a way that $\left\{P_{i j}(t)\right\}$ transforms the sequence with 1 in the $m$ th place and 0 elsewhere into the sequence whose $k$ th term is $P_{m k}(t)$. We will use letters like $v$ to denote a sequence, $T$ to denote a particular matrix and $T(v)$ to denote the sequence $v$ transformed by the matrix $T$.

Our first step will be to show that $P_{11}(t)$ has bounded variation in some interval (say from 0 to $t_{0}$ ). To do this we will estimate $\sum_{i=0}^{N-1}\left|P_{11}\left(i t_{0} / N\right)-P_{11}\left((i+1) t_{0} / N\right)\right|$ for a fixed integer $N$. The estimate will turn out to be independent of $N$. To simplify notation we will let $T=\left\{P_{i j}\left(t_{0} / N\right)\right\}$ and let $f_{i}=P_{11}\left(i t_{0} / N\right)$.

We will first define a sequence of vectors (or sequences) $v_{i}$. $v_{0}$ will be the sequence with 1 in the first place and 0 elsewhere. Let us de-

1 This research was supported in part by the United States Air Force under contract AF49(638)-265, monitored by the Office of Scientific Research.

2 This condition has been eliminated by Professor Jurkat.

3 I wish to thank Professor Chung for valuable aid and encouragement in the preparation of this paper. 
denote by $v^{*}$ the sequence whose first term is 0 and which agrees with $v$ everywhere else. Define $v_{i+1}=\left(T\left(v_{i}\right)\right)^{*}$. We then have

$$
T^{s}\left(v_{0}\right)=\sum_{i=0}^{s} f_{s-i} v_{i} \text {. }
$$

This is easily verified by induction (note that the first coordinate of $T^{s}\left(v_{0}\right)=f_{s}$ by definition). We will define a sequence of positive real numbers $\beta_{i} . \beta_{0}=1-f_{1}$ and $\beta_{i}(i \geqq 1)$ is the first coordinate of $T\left(v_{i}\right)$. The following formula is also easy to check.

$$
f_{s+1}-f_{s}=-f_{s} \beta_{0}+\sum_{i=1}^{s} f_{s-i} \beta_{i} .
$$

(We must interpret $\sum_{i=1}^{0}$ as 0 ). Rewriting (2) we get

$$
f_{s+1}-f_{s}=f_{s} \sum_{i=1}^{s} \beta_{i}-f_{s} \beta_{0}+\sum_{i=1}^{s}\left(f_{s-i}-f_{s}\right) \beta_{i} \text {. }
$$

3) $\sum_{s=0}^{N-1}\left|f_{s}-f_{s+1}\right| \leqq \sum_{s=0}^{N-1}\left|f_{s} \sum_{i=1}^{s} \beta_{i}-f_{s} \beta_{0}\right|+\sum_{s=0}^{N-1} \sum_{i=1}^{8}\left|f_{s-i}-f_{s}\right| \beta_{i}$.

$$
\sum_{s=0}^{N-1} \sum_{i=1}^{s}\left|f_{s-i}-f_{s}\right| \beta_{i} \leqq\left(\sum_{s=0}^{N-1}\left|f_{s}-f_{s+1}\right|\right)\left(\sum_{i=1}^{N-1} i \beta_{i}\right) .
$$

To see (4) note that

$$
\sum_{s=0}^{N-1} \sum_{i=1}^{\dot{1}}\left|f_{s-i}-f_{s}\right| \beta_{i}=\sum_{j=1}^{N-1} \sum_{k=j}^{N-1}\left|f_{k-j}-f_{k}\right| \beta_{j}
$$

and

$$
\sum_{k=i}^{N-1}\left|f_{k-j}-f_{k}\right| \leqq i \sum_{s=0}^{N-1}\left|f_{s}-f_{s+1}\right| .
$$

From (3) and (4) we get

$$
\begin{array}{r}
\sum_{s=0}^{N-1}\left|f_{s}-f_{s+1}\right| \leqq\left(\sum_{s=0}^{N-1}\left|f_{s}-f_{s+1}\right|\right)\left(\sum_{i=1}^{N-1} i \beta_{i}\right) \\
+\sum_{s=0}^{N-1}\left|f_{s} \sum_{i=1}^{\dot{S}} \beta_{i}-f_{s} \beta_{0}\right| .
\end{array}
$$

If we now assume that the $t_{0}$ we used in defining $T$ has the property that $P_{11}(t)>3 / 4$ for all $t<t_{0}$ we will be able to show that both $\sum_{i=1}^{N-1} i \beta_{i}$ and $\sum_{s=0}^{N-1}\left|f_{s} \sum_{i=1}^{s} \beta_{i}-f_{s} \beta_{0}\right|$ are $<1 / 2$. This and (5) will then immediately imply that $\sum_{s=0}^{N-1}\left|f_{s}-f_{s+1}\right|<1$ and, since $P_{11}(t)$ is 
continuous and our estimate does not depend on $N$, that the variation of $P_{11}(t)\left(t<t_{0}\right)$ is $\leqq 1$. To get $\sum_{i=1}^{N-1} i \beta_{i}<1 / 2$ we note first that $\sum_{i=1}^{N-1} i \beta_{i}<\sum_{i=1}^{N}\left|v_{i}\right|(|v|=$ sum of the absolute values of the coordinates of $v)$ since $\beta_{i}=\left|v_{i}\right|-\left|v_{i+1}\right|$. Next we show that $\sum_{i=1}^{N}\left|v_{i}\right|$ $<1 / 2$. $T^{N}\left(v_{0}\right)=f_{N} v_{0}+\sum_{i=1}^{N} f_{N-i} v_{i}$ and since row sums $=1$, $\sum_{i=1}^{N} f_{N-i}\left|v_{i}\right|=1-f_{N}<1 / 4$. Each of the $f_{N-i}>1 / 2$ so $\sum_{i=1}^{N}\left|v_{i}\right|$ $<1 / 2$. $\sum_{s=0}^{N-1}\left|f_{s} \sum_{i=1}^{s} \beta_{i}-f_{s} \beta_{0}\right|<1 / 2$ because $\left|\beta_{0}-\sum_{i=1}^{s} \beta_{i}\right|=\left|v_{s+1}\right|$.

We now know that $P_{11}(t)$ has variation $<1$ in a certain interval about 0 . The following argument shows that the variation of $P_{1 j}(t) \leqq 4$ in the same interval.

$$
\begin{aligned}
T^{s+1}\left(v_{0}\right)-T^{s}\left(v_{0}\right) & =\sum_{i=0}^{s+1}\left(f_{s+1-i}-f_{s-i}\right) v_{i}, \quad\left(f_{-1}=0\right) \\
\sum_{s=0}^{N-1}\left|T^{s+1}\left(v_{0}\right)-T^{s}\left(v_{0}\right)\right| & \leqq \sum_{i=0}^{N} \sum_{s=i-1}^{N-1}\left|\left(f_{s+1-i}-f_{s-i}\right) v_{i}\right| \\
& \leqq \sum_{i=0}^{N} 2\left|v_{i}\right| \leqq 4 .
\end{aligned}
$$

The remainder of the proof follows a suggestion of K. L. Chung. ${ }^{4}$ Functions of bounded variation have a finite derivative almost everywhere and we can therefore pick a $t_{1}<t_{0}$ such that $P_{1 j}(t)$ has a derivative at $t_{1}$ for all $j$. For an arbitrary $t_{2}$ the existence of a derivative for $P_{1 i}\left(t_{1}+t_{2}\right)(i=1 \cdots \infty)$ follows from the fact that

$$
\frac{P_{1 i}\left(t_{1}+t_{2}\right)-P_{1 i}\left(t_{1}+t_{2}+\alpha\right)}{\alpha}=\sum_{k=1}^{\infty} \frac{P_{1 k}\left(t_{1}\right)-P_{1 k}\left(t_{1}+\alpha\right)}{\alpha} P_{k i}\left(t_{2}\right)
$$

and the following lemma: given $\epsilon$ there exists an integer $K$ such that

$$
\sum_{j=K}^{\infty} \frac{\left|P_{1 j}\left(t_{1}\right)-P_{1 j}\left(t_{1}+\alpha\right)\right|}{\alpha}<\epsilon, \quad \frac{t_{1}}{4}>\alpha>0 .
$$

We conclude by proving (7). For a given $\alpha<t_{1} / 4$ we will pick a $t_{0}^{\prime}$ between $t_{1}$ and $t_{1} / 2$ and an integer $N$ such that $t_{0}^{\prime} / N=\alpha$ and we will define $T$ and $v_{i}$ as before, except that we will use $t_{0}^{\prime}$ instead of $t_{0}$.

It is easy to show that given $\epsilon_{1}$ (we will pick $\epsilon_{1}$ to be $<(1 / 8) \epsilon \cdot t_{1} / 2$ $\cdot 1 / 2)$ there is a $K_{1}$ such that $\sum_{j=K_{1}}^{\infty} P_{1 j}(t)<\epsilon_{1}$ for all $t<t_{1}$. We then have $\sum_{i=1}^{N}\left|v_{i}^{K_{1}}\right|<2 \epsilon_{1}\left(\left|v_{i}^{K_{1}}\right|\right.$ is the sum of the absolute values of the terms of $v_{i}$ with index $\left.\geqq K_{1}\right)$. The same argument as the one used in (6) shows

4 The original proof did not make use of the theorem that functions of bounded variation have derivatives almost everywhere and was very much longer. Professor Chung's idea also gives $P_{1 i}^{\prime}\left(t_{1}+t_{2}\right)=\sum_{k} P_{1 k}\left(t_{1}\right) P_{k i}^{\prime}\left(t_{2}\right)$. Professor Chung has also proved (in a different way) that $P_{1 i}^{\prime}\left(t_{1}+t_{2}\right)=\sum_{k} P_{1 k}\left(t_{1}\right) P_{k i}^{\prime}\left(t_{2}\right)$. 


$$
\sum_{s=1}^{N-1} \sum_{j=K_{1}}^{\infty}\left|P_{1 j}((s+1) \alpha)-P_{1 j}(s \alpha)\right|<4 \epsilon_{1} .
$$

There are at least $(N-1) / 2$ integers $s$ such that

$$
\sum_{j=K_{1}}^{\infty}\left|P_{1 j}((s+1) \alpha)-P_{1 j}(s \alpha)\right|<8 \epsilon_{1} \frac{1}{N}
$$

and for one of these, call it $r$,

$$
\sum_{j=1}^{K_{1}}\left|P_{1 j}((r+1) \alpha)-P_{1 j}(r \alpha)\right|<\frac{8}{N} .
$$

This follows from (6). We now pick $\epsilon_{2}$ (make it $<\epsilon \cdot(1 / 8) K_{1} \cdot t_{1 / 2} \cdot 1 / 2$ ). There is a $K>K_{1}$ such that

$$
\begin{aligned}
& \sum_{j=K}^{\infty} P_{i j}(t)<\epsilon_{2} \quad \text { for all } t<t_{1} \text { and } i \leqq K_{1}, \\
& \sum_{j=K}^{\infty} \mid P_{1 j}\left(t_{1}\right)- P_{1 j}\left(t_{1}+\alpha\right) \mid \\
& \leqq \sum_{m=K}^{\infty} \sum_{j=1}^{\infty}\left|P_{1 j}(r \alpha)-P_{1 j}(r+1) \alpha\right| P_{j m}\left(t_{1}-r \alpha\right) \\
&= \sum_{m=K}^{\infty} \sum_{j=K_{1}+1}^{\infty}\left|P_{1 j}(r \alpha)-P_{1 j}((r+1) \alpha)\right| P_{j m}(t-r \alpha) \\
&+\sum_{m=K}^{\infty} \sum_{j=1}^{K_{1}}\left|P_{1 j}(r \alpha)-P_{1 j}((r+1) \alpha)\right| P_{j m}\left(t_{1}-r \alpha\right) .
\end{aligned}
$$

The first term of this last expression is $<8 \epsilon_{1} \cdot 1 / N$ by (9), $\mid P_{1 j}(r \alpha)-P_{1 j}\left((r+1) \alpha \mid<8 / N\right.$ by $(10)$ and $\sum_{m=K}^{\infty} P_{j m}\left(t_{1}-r \alpha\right)<\epsilon_{2}$ for each $j<K_{1}$ by (11). Hence the second term is $<8 / N \cdot \epsilon_{2} \cdot K_{1}$.

This finishes the proof of the lemma.

\section{BIBLIOGRAPHY}

1. D. G. Austin, On the existence of the derivative of Markoff transition probability functions, Proc. Nat. Acad. Sci. U. S. A. vol. 41 (1941) pp. 224-226.

2. - Some differentiability properties of Markoff transition probability functions, Proc. Amer. Math. Soc. vol. 7 (1956) pp. 751-761.

3. J. L. Doob, Topics in the theory of Markoff chains, Trans. Amer. Math. Soc. vol. 52 (1942) pp. 37-64.

4. A. N. Kolmogoroff, On the differentiability of transition probabilities in stationary Markov processes with a denumerable number of states, Moskov. Gos. Univ. Ux. Zap. Mat. vol. 148 (1951) pp. 53-59 (Russian).

UNIVERSITY OF WISCONSIN 